\title{
Review Article \\ Compatibility Conditions and the Convolution of Functions and Generalized Functions
}

\author{
Andrzej Kamiński and Svetlana Mincheva-Kaminska \\ Faculty of Mathematics and Natural Sciences, University of Rzeszów, Profesora Pigonia 1, 35-310 Rzeszów, Poland \\ Correspondence should be addressed to Andrzej Kamiński; akaminsk@ur.edu.pl
}

Received 23 July 2013; Accepted 6 September 2013

Academic Editor: Henryk Hudzik

Copyright (C) 2013 A. Kamiński and S. Mincheva-Kaminska. This is an open access article distributed under the Creative Commons Attribution License, which permits unrestricted use, distribution, and reproduction in any medium, provided the original work is properly cited.

\begin{abstract}
The paper is a review of certain existence theorems concerning the convolution of functions, distributions, and ultradistributions of Beurling type with supports satisfying suitable compatibility conditions. The fact that some conditions are essential for the existence of the convolution in the discussed spaces follows from earlier results and the proofs given at the end of this paper.
\end{abstract}

In memory of Professor Jan Mikusinski on the 100th anniversary of his birthday

\section{Introduction}

The convolution and its various generalizations play a very important role in the classical and abstract analysis as well as in other fields of mathematics, in particular in the theory of distributions (see [1-7]), ultradistributions (see [3, 4, 8-18]), hyperfunctions (see [19-21]), and other generalized functions considered for various spaces, subspaces, and approaches. The notion of convolution is a starting point in algebraic approaches to certain generalized functions: the convolution algebra of continuous functions on $[0, \infty)$ extends, due to the Titchmarsh theorem, to the field of Mikusiński operators (see [22-25]) which are generalized functions of another type than Schwartz distributions, while Boehmians stand for a common generalization of regular Mikusiński operators of Boehme (see [26]), Schwartz distributions, and other classes of generalized functions on the real line (see [27-31]). An important part of investigations connected with the convolution is the study of convolution operators and convolution semigroups for various spaces of functions and generalized functions (see, e.g., [32-37]). Therefore the problems concerning the existence of the convolution in various spaces of functions and generalized functions are crucial.
The theory developed by Colombeau (see [38]; see also [39]) and his followers (see, e.g., [40-43]) has led to constructions of algebras of new generalized functions related to the distributions and other classical generalized functions due to certain quotient procedures; consequently the algebras of new generalized functions are closed with respect to multiplication as well as other nonlinear operations. However the problem of existence of the product and the convolution of distributions and other generalized functions in the standard sense, without using Colombeau's approach, remains important. We will analyse in this paper the existence of the convolution of distributions and tempered distributions on $\mathbb{R}^{d}$ meant in the classical sense of general equivalent definitions introduced independently by several authors (see $[2,44-50])$. Also the existence of the convolution of ultradistributions and tempered ultradistributions of Beurling type introduced in $[18,51]$ (see also [52]), corresponding to the above mentioned general definitions of the convolution of distributions and tempered distributions, will be discussed. The convolution of ultradistributions of Roumieu type will be not considered in this paper; for recently obtained results concerning the convolution of Roumieu ultradistributions we refer to the paper [53]. 
There exist various sufficient conditions guaranteeing existence of the convolution in various spaces of functions and generalized functions. Some of the conditions are given in the form of suitable assumptions concerning their growth at infinity, but there are conditions of another type expressed in terms of supports of given (generalized) functions. We will call them after Mikusiński (see [54], and [7, p. 124-127]) compatibility conditions. We mean the support of a given function (generalized function) in the standard way as the smallest closed set in $\mathbb{R}^{d}$ outside which the function (generalized function) vanishes almost everywhere (everywhere). For modifications of the notion of support in case of distributions and tempered distributions see [7, p. 241] and [55].

We recall in this paper various versions of compatibility conditions imposed on supports of functions, distributions, and ultradistributions of Beurling type which guarantee that the convolution in the respective spaces exists (see $[5,7,54,56-59]$; see also [60-63]). In Section 7, we prove some inverse theorems which mean that the considered compatibility conditions are optimal for the existence of the convolution in some spaces in terms of supports; that is, the conditions cannot be relaxed. We also mention new cases of compatibility of supports in which the convolution exists in the considered spaces. In particular, we show examples of the so-called spiral sets in $\mathbb{R}^{d}$ with the property that the convolutions of functions, distributions, ultradistributions having supports contained in such sets always exist in the respective spaces, though the sets are unbounded in each direction of $\mathbb{R}^{d}$.

\section{Preliminaries}

We will use the standard multidimensional notation concerning $\mathbb{R}^{d}$ and $\mathbb{N}_{0}^{d}$, using traditional symbols even in case they are formally inconsistent, because the proper meaning is easily seen from the context; for example, $|x|$ denotes the Euclidean norm of $x$ in $\mathbb{R}^{d}$ and

$$
\begin{aligned}
\langle x\rangle:=\left(1+|x|^{2}\right)^{1 / 2}= & \left(1+\xi_{1}^{2}+\cdots+\xi_{d}^{2}\right)^{1 / 2}, \\
& x=\left(\xi_{1}, \ldots, \xi_{d}\right) \in \mathbb{R}^{d},
\end{aligned}
$$

but we write also $|k|:=\kappa_{1}+\cdots+\kappa_{d}$ for $k=\left(\kappa_{1}, \ldots, \kappa_{d}\right) \in \mathbb{N}_{0}^{d}$, though $\mathbb{N}_{0}^{d} \subset \mathbb{R}^{d}$.

The standard notation will be also used for the known spaces of (complex-valued) functions on $\mathbb{R}^{d}: L^{r}\left(\mathbb{R}^{d}\right)$ for $r \in$ $[1, \infty]$ (with the norm denoted by $\left.\|\cdot\|_{r}\right), L_{\mathrm{loc}}^{1}\left(\mathbb{R}^{d}\right), \mathscr{C}\left(\mathbb{R}^{d}\right)$, $\mathscr{C}^{\infty}\left(\mathbb{R}^{d}\right), \mathscr{E}\left(\mathbb{R}^{d}\right), \mathscr{D}\left(\mathbb{R}^{d}\right), \mathscr{D}_{K}\left(\mathbb{R}^{d}\right), \mathcal{S}\left(\mathbb{R}^{d}\right)$, and the known (sub) spaces of distributions on $\mathbb{R}^{d}: \mathscr{C}^{\prime}\left(\mathbb{R}^{d}\right), \mathscr{D}^{\prime}\left(\mathbb{R}^{d}\right)$, and $\mathcal{S}^{\prime}\left(\mathbb{R}^{d}\right)$ (cf. $\left.[1,7]\right)$.

For a set $E \subseteq \mathbb{R}^{d}$ we denote $E^{c}:=\mathbb{R}^{d} \backslash E$. It will be convenient to use the following notation for $E \subseteq \mathbb{R}^{d}$ and a function $\phi$ on $\mathbb{R}^{d}$ :

$$
\begin{aligned}
& E^{\Delta}:=\left\{(x, y) \in \mathbb{R}^{2 d}: x+y \in E\right\}, \\
& \phi^{\Delta}(x, y):=\phi(x+y), \quad x, y \in \mathbb{R}^{d} .
\end{aligned}
$$

Clearly, for any $E \subseteq \mathbb{R}^{d}$, the set $E^{\Delta}$ is bounded (compact) in $\mathbb{R}^{2 d}$ if and only if $E=\emptyset$. Therefore if $\phi \in \mathscr{D}\left(\mathbb{R}^{d}\right)$, then $\phi^{\Delta} \in$ $\mathscr{C}^{\infty}\left(\mathbb{R}^{2 d}\right)$, but $\phi^{\Delta} \in \mathscr{D}\left(\mathbb{R}^{2 d}\right)$ only in case $\phi=0$. Moreover, the symbol $\sqsubset \mathbb{R}^{d}$ will be used in the following sense:

$$
E \subset \mathbb{R}^{d} \quad \text { whenever } E \text { is a compact subset of } \mathbb{R}^{d} .
$$

The words "measure" and "measurable" concerning the sets in $\mathbb{R}^{d}$ are considered in the sense of Lebesgue; the Lebesgue measure of a given Lebesgue measurable set $A \subseteq \mathbb{R}^{d}$ will be denoted by $\ell^{d}(A)$. If $A \subseteq \mathbb{R}^{d}$ is a measurable set, we use the following notation:

$$
|A|_{\infty}:=\sup \operatorname{ess}\{|x|: x \in A\} .
$$

That is, $|A|_{\infty}=\inf \Lambda(A)$ in case $\Lambda(A) \neq \emptyset$ and $|A|_{\infty}=\infty$ in case $\Lambda(A)=\emptyset$, where $\Lambda(A):=\left\{\alpha>0: \ell^{d}\left(X_{\alpha}\right)=0\right\}$ with $X_{\alpha}:=\{x \in A:|x|>\alpha\}$. Clearly,

$$
|A|_{\infty}=0 \quad \text { iff } \ell^{d}(A)=0 .
$$

We call a measurable set $A \subset \mathbb{R}^{d}$ positively bounded in $\mathbb{R}^{d}$ whenever $0<|A|_{\infty}<\infty$. An element $x$ of a measurable set $A \in \mathbb{R}^{d}$ is called a massive point of the set $A$ if $\ell^{d}(A \cap U(x))>0$ for each open neighbourhood $U(x)$ of $x$ in $\mathbb{R}^{d}$.

A measurable function $F$ on $\mathbb{R}^{d}$ is called slowly increasing (resp., rapidly decreasing) if $|F(x)| \leq P(x)$ (resp., $|P(x) F(x)| \leq$ $c(P))$ for a certain polynomial $P$ on $\mathbb{R}^{d}$ (resp., for every polynomial $P$ and some positive constant $c(P)$ ) and for almost all $x \in \mathbb{R}^{d}$. The sets of all measurable slowly increasing and rapidly decreasing functions on $\mathbb{R}^{d}$ will be denoted by $\mathscr{P}\left(\mathbb{R}^{d}\right)$ and $\mathscr{R}\left(\mathbb{R}^{d}\right)$, respectively.

In our considerations concerning the convolution in the spaces $\mathscr{D}^{\prime\left(M_{p}\right)}\left(\mathbb{R}^{d}\right)$ of ultradistributions and $\mathcal{S}^{\prime\left(M_{p}\right)}\left(\mathbb{R}^{d}\right)$ of tempered ultradistributions of Beurling type (the respective definitions and notation are introduced in Section 4) we always assume that a given sequence $\left(M_{p}\right)=\left(M_{p}\right)_{p \in \mathbb{N}_{0}}$ of positive numbers satisfies the following three conditions (see $[10-12,18,51,52,59])$ :

$$
\begin{aligned}
& \text { (M.1) } M_{p}^{2} \leq M_{p-1} M_{p+1}, p \in \mathbb{N} ; \\
& \text { (M.2) } M_{p} \leq a b^{p} M_{q} M_{p-q}, p, q \in \mathbb{N}, 0 \leq q \leq p ; \\
& \text { (M.3) } \sum_{p=q+1}^{\infty} M_{p-1} M_{p}^{-1} \leq a q M_{q} M_{p}^{-1}, q \in \mathbb{N},
\end{aligned}
$$

where $a>0$ and $b>0$ are some constants. We extend the sequence $\left(M_{p}\right)_{p \in \mathbb{N}_{0}}$ to its multidimensional version $\left(M_{k}\right)_{k \in \mathbb{N}_{0}^{d}}$ as follows:

$$
M_{k}:=M_{\kappa_{1}+\cdots+\kappa_{d}}, \quad k=\left(\kappa_{1}, \ldots, \kappa_{d}\right) \in \mathbb{N}_{0}^{d} .
$$

By the associated function for the sequence $\left(M_{p}\right)$ we mean the function on $[0, \infty)$ given by

$$
M(t):=\sup _{p \in \mathbb{N}_{0}} \log _{+}\left(\frac{t^{p}}{M_{p}}\right), \quad t>0,
$$

where $\log _{+}:=\max \{\log t, 0\}$ and $M(0):=0$. 


\section{Convolution of Functions and Distributions}

Definition 1. Let $F$ and $G$ be Lebesgue measurable functions on $\mathbb{R}^{d}$. For a given $x \in \mathbb{R}^{d}$ one defines

$$
(F * G)(x):=\int_{\mathbb{R}^{d}} F(x-t) G(t) d t,
$$

saying that $(F * G)(x)$ exists, whenever the function under the integral sign in (8) is Lebesgue integrable as a function of $t$ for the fixed $x$. If $(F * G)(x)$ exists or, equivalently, $(|F| *$ $|G|)(x)$ exists, for all (almost all) $x \in \mathbb{R}^{d}$, then one says that the convolution $F * G$ exists everywhere (almost everywhere) on $\mathbb{R}^{d}$.

Definition 2. Let $F, G \in L_{\text {loc }}^{1}\left(\mathbb{R}^{d}\right)$. One says that the convolution $F * G$ exists in $L_{\text {loc }}^{1}$ if $F * G$ exists almost everywhere on $\mathbb{R}^{d}$ and $|F| *|G| \in L_{\text {loc }}^{1}\left(\mathbb{R}^{d}\right)$.

Let us list some classical results concerning the existence in $L_{\text {loc }}^{1}$ of the convolution of functions of certain classes:

(a) if $F, G \in L^{1}\left(\mathbb{R}^{d}\right)$, then $F * G$ exists in $L_{\text {loc }}^{1}$ and $F * G \epsilon$ $L^{1}\left(\mathbb{R}^{d}\right)$;

(b) if $F \in L^{p}\left(\mathbb{R}^{d}\right)$ and $G \in L^{q}\left(\mathbb{R}^{d}\right)$, where $p, q \in[1, \infty)$ such that $1 / p+1 / q>1$, then $F * G$ exists in $L_{\text {loc }}^{1}$ and $F * G \in L^{r}\left(\mathbb{R}^{d}\right)$, where $r=(1 / p+1 / q-1)^{-1}$ (Young);

(c) if $F \in \mathscr{P}\left(\mathbb{R}^{d}\right)$ and $G \in \mathscr{R}\left(\mathbb{R}^{d}\right)$, then $F * G$ exists in $L_{\text {loc }}^{1}$ and $F * G \in \mathscr{P}\left(\mathbb{R}^{d}\right)$.

However, in general, the existence of the convolution of functions in $L_{\text {loc }}^{1}$ does not imply any restriction of growth of the convolution at infinity. For instance, the convolution of two measurable slowly increasing functions may exist in $L_{\text {loc }}^{1}$, but their convolution may be a function of arbitrarily fast increase at infinity.

The following result, much stronger than one may expect, was proved in $[56,57]$ (see also $[58,63]$ ).

Theorem 3. Let $\Phi$ be an arbitrary continuous (complexvalued) function on $\mathbb{R}^{d}$. There exists a nonnegative $C^{\infty}$ function $\varphi$ on $\mathbb{R}^{d}$ such that

$$
\lim _{|x| \rightarrow \infty} \varphi(x)=0
$$

the convolution $\varphi * \varphi$ exists everywhere in $\mathbb{R}^{d}$, and the following inequality is satisfied:

$$
(\varphi * \varphi)(x)>|\Phi(x)| \quad \forall x \in \mathbb{R}^{d} .
$$

Moreover, the function $\varphi$ can be constructed in such a way that its support is compatible with itself (see Definition 16).

Let us underline that inequality (10) in the assertion of Theorem 3 is satisfied for each $x \in \mathbb{R}^{d}$; the convergence in (9) can be slow: the faster the increase of the function $\Phi$ (i.e., the required increase of the convolution $\varphi * \varphi$ ) is at infinity the slower the approach of $\varphi$ to 0 is as $|x|$ tends to $\infty$ (for details of the construction see $[56,57,63])$.

Theorem 3 has a general value. From the theorem one may easily see, for various spaces of functions or generalized functions and their various subspaces, that the convolution in the sense of the considered space leads out of the examined subspace. This concerns, in particular, the convolution in $\mathscr{D}^{\prime}$ and in $\mathscr{D}^{\prime\left(M_{p}\right)}$ (see Definitions 5 and 12) of elements of the subspaces $\mathcal{S}^{\prime}\left(\mathbb{R}^{d}\right)$ and $\mathcal{S}^{\prime\left(M_{p}\right)}\left(\mathbb{R}^{d}\right)$ of the spaces $\mathscr{D}^{\prime}\left(\mathbb{R}^{d}\right)$ and $\mathscr{D}^{\prime\left(M_{p}\right)}\left(\mathbb{R}^{d}\right)$ of Schwartz distributions and Beurling ultradistributions, respectively (see the definitions of the spaces, given in this and in the next section, and the comments at the end of this section).

Let us shortly recall (see [1]; see also, e.g., [5-7]) that distributions are elements of the strong dual $\mathscr{D}^{\prime}\left(\mathbb{R}^{d}\right)$ of the basic space $\mathscr{D}\left(\mathbb{R}^{d}\right)$ of test functions, that is, linear continuous functionals on the space $\mathscr{D}\left(\mathbb{R}^{d}\right)$ defined as the inductive limit:

$$
\mathscr{D}\left(\mathbb{R}^{d}\right):=\operatorname{ind}_{K \subset \subset \mathbb{R}^{d}} \lim _{K}\left(\mathbb{R}^{d}\right),
$$

where the symbol $K \sqsubset \sqsubset \mathbb{R}^{d}$ means here that $K$ are compact sets growing up to $\mathbb{R}^{d}$. Recall that $\mathscr{D}_{K}\left(\mathbb{R}^{d}\right)$, for a fixed $K \sqsubset$ $\mathbb{R}^{d}$, means the space of all $C^{\infty}$ functions on $\mathbb{R}^{d}$ whose support is contained in $K$, with the topology given by the family $\left\{\|\cdot\|_{K, m}: m \in \mathbb{N}_{0}\right\}$ of the norms defined as follows:

$$
\begin{array}{r}
\|\varphi\|_{K, m}:=\sup \left\{\left|\varphi^{(k)}(x)\right|: x \in K, k \in \mathbb{N}_{0}^{d},|k| \leq m\right\}, \\
m \in \mathbb{N}_{0} .
\end{array}
$$

Locally integrable functions on $\mathbb{R}^{d}$ may be treated as distributions (so-called regular distributions), because the space $L_{\text {loc }}^{1}\left(\mathbb{R}^{d}\right)$ can be naturally embedded into $\mathscr{D}^{\prime}\left(\mathbb{R}^{d}\right)$. The known structural theorem describes every distribution as a derivative (in the distributional sense) locally, that is, on each relatively compact set in $\mathbb{R}^{d}$, of finite order of a continuous function (see [1, 5-7]).

Recall that tempered distributions are elements of the dual $\delta^{\prime}\left(\mathbb{R}^{d}\right)$ of the complete metric space $\delta\left(\mathbb{R}^{d}\right)$ of rapidly decreasing $C^{\infty}$ functions, that is, of all $\varphi \in \mathscr{C}^{\infty}\left(\mathbb{R}^{d}\right)$ such that

$$
\sigma_{m}(\varphi):=\max \left\{\left\|\langle\cdot\rangle^{i} \varphi^{(k)}\right\|_{\infty}: i, k \in \mathbb{N}_{0}^{d},|i|,|k| \leq m\right\}<\infty
$$

for all $m \in \mathbb{N}_{0}$, where $\langle\cdot\rangle$ is defined by (1), with the metric topology induced by the family $\left\{\sigma_{m}: m \in \mathbb{N}_{0}\right\}$ of the norms defined in (13). Since $\mathscr{D}\left(\mathbb{R}^{d}\right)$ is dense in $\mathcal{S}\left(\mathbb{R}^{d}\right)$, the space $\delta^{\prime}\left(\mathbb{R}^{d}\right)$ of tempered distributions can be naturally embedded into $\mathscr{D}^{\prime}\left(\mathbb{R}^{d}\right)$. The known structural theorem describes each tempered distribution as a derivative (in the distributional sense) of finite order of a continuous slowly increasing function on $\mathbb{R}^{d}$ (see [1-7]).

There are several general definitions of the convolution in $\mathscr{D}^{\prime}$ of Schwartz distributions given consecutively by Chevalley [2], Schwartz [44], Shiraishi [45], and Vladimirov [46] (see also [47-50, 63-72]). 
Most of the mentioned definitions are equivalent (for details see, e.g., [50]). We will recall only one of them, the sequential definition of Vladimirov [46] (see also [6]), based on the notion of strong approximate unit.

Definition 4. A sequence $\left(\eta_{n}\right)$ of elements of $\mathscr{D}\left(\mathbb{R}^{d}\right)$ is said to be a strong approximate unit on $\mathbb{R}^{d}$ if for every $K \sqsubset \mathbb{R}^{d}$ there exists an $n_{0} \in \mathbb{N}$ such that $\eta_{n}(x)=1$ for $x \in K$ and $n \geq n_{0}$ (hence $\eta_{n} \rightarrow 1$ in $\mathscr{E}\left(\mathbb{R}^{d}\right)$ as $n \rightarrow \infty$ ) and, in addition,

$$
\sup _{n \in \mathbb{N}}\left\|\eta_{n}^{(k)}\right\|_{\infty}<\infty \quad \text { for every } k \in \mathbb{N}_{0}^{d} .
$$

One denotes the set of all strong approximate units on $\mathbb{R}^{d}$ by $\bar{U}\left(\mathbb{R}^{d}\right)$.

Definition 5. For given $S, T \in \mathscr{D}^{\prime}\left(\mathbb{R}^{d}\right)$ the convolution $S * T$ in $\mathscr{D}^{\prime}$ is defined by

$$
\langle S * T, \varphi\rangle:=\lim _{n \rightarrow \infty}\left\langle S \otimes T, \eta_{n} \varphi^{\Delta}\right\rangle, \quad \varphi \in \mathscr{D}\left(\mathbb{R}^{d}\right),
$$

whenever the above limit exists for every strong approximate unit $\left(\eta_{n}\right) \in \overline{\mathbb{U}}\left(\mathbb{R}^{2 d}\right)$ and $\varphi \in \mathscr{D}\left(\mathbb{R}^{d}\right)$. One says then that the convolution $S * T$ exists in $\mathscr{D}^{\prime}$.

By the Lebesgue theorem, if $F, G \in L_{\text {loc }}^{1}\left(\mathbb{R}^{d}\right)$ and the convolution $F * G$ exists in $L_{\text {loc }}^{1}$, then it exists in $\mathscr{D}^{\prime}$ and represents a regular distribution in $\mathscr{D}^{\prime}\left(\mathbb{R}^{d}\right)$ (see $[6$, p. 63]).

Analogously to and independently of Definition 5, one may define the convolution in $\delta^{\prime}$ of tempered distributions in various ways (see, e.g., $[45,49,50]$ and other references given earlier). Again, we present below only one of several equivalent definitions of the convolution in $\mathcal{S}^{\prime}$, namely, the respective counterpart of the aforementioned sequential definition of Vladimirov (cf. $[6,46])$.

Definition 6. For given $S, T \in \mathcal{S}^{\prime}\left(\mathbb{R}^{d}\right)$ one defines the convolution $S * T$ in $\mathcal{S}^{\prime}$ by

$$
\langle S * T, \psi\rangle:=\lim _{n \rightarrow \infty}\left\langle S \otimes T, \eta_{n} \psi^{\Delta}\right\rangle, \quad \psi \in \mathcal{S}\left(\mathbb{R}^{d}\right)
$$

whenever the above limit exists for every strong approximate unit $\left(\eta_{n}\right) \in \bar{U}\left(\mathbb{R}^{2 d}\right)$ and $\psi \in \mathcal{S}\left(\mathbb{R}^{d}\right)$. One says then that the convolution $S * T$ exists in $\mathcal{S}^{\prime}$.

In [45], Shiraishi posed the problem of whether the assumption that the convolution $S * T$ of two tempered distributions $S, T \in \mathcal{S}^{\prime}\left(\mathbb{R}^{d}\right)$ exists in $\mathscr{D}^{\prime}$ implies the existence of the convolution $S * T$ in $\mathcal{S}^{\prime}$ (in particular, whether $S * T \in$ $\left.\mathcal{S}^{\prime}\left(\mathbb{R}^{d}\right)\right)$.

The negative answer to Shiraishi's problem follows directly from Theorem 3, proved by Kamiński in [56, 57] (see also $[58,63])$ and presented in Professor Jan Mikusiński's seminar in Katowice in 1971 and during the international conference on generalized functions in Rostock in 1972. This also follows from a result obtained independently by Dierolf and Voigt and published in [49]. Dierolf and Voigt constructed in [49] two tempered measures $S$ and $T$, concentrated on a countable set in $\mathbb{R}^{1}$, such that the convolution $S * T$ exists in $\mathscr{D}^{\prime}$, but $f * g \notin \mathcal{S}^{\prime}\left(\mathbb{R}^{1}\right)$.

Theorem 3 is much stronger than the result of Dierolf and Voigt (it supplies counterexamples concerning the growth of the convolution in various spaces of functions and generalized functions).

To get the negative answer to Shiraishi's problem it is enough to take in Theorem 3 for $\Phi$ the function defined by

$$
\Phi(x):=\exp x=\exp \left(\xi_{1}+\cdots+\xi_{d}\right), \quad x=\left(\xi_{1}, \ldots, \xi_{d}\right) \in \mathbb{R}^{d}
$$

(or any continuous function of even faster increase). Clearly, $\Phi$ represents a distribution which is not tempered, but from Theorem 3 it follows that there exists a bounded (even vanishing at infinity) $C^{\infty}$ function $\varphi$ on $\mathbb{R}^{d}$ representing a tempered distribution for which the convolution $\varphi * \varphi$ exists everywhere and exceeds everywhere on $\mathbb{R}^{d}$ the function $\Phi$. Consequently, $\varphi * \varphi$ represents a distribution but not a tempered distribution.

\section{Convolution of Beurling Ultradistributions}

We will recall the definitions of the space of $\mathscr{D}^{\prime\left(M_{p}\right)}\left(\mathbb{R}^{d}\right)$ of Beurling ultradistributions (see $[9-12,18,51]$ ) and the space $\mathcal{S}^{\prime\left(M_{p}\right)}\left(\mathbb{R}^{d}\right)$ of Beurling tempered ultradistributions (see $[18,52,59,73])$ as well as the corresponding structural theorems characterizing elements of these spaces for a fixed numerical sequence $\left(M_{p}\right)$ satisfying conditions (M.1)-(M.3) (see Section 2). Ultradistributions of Roumieu type (see [8]) are not discussed in this paper.

We start by defining Beurling spaces of ultradifferentiable functions. For a given $h>0$ and a regular compact subset $K$ of $\mathbb{R}^{d}$ (see [10-12]), we define the space $\mathscr{D}_{K, h}^{\left(M_{p}\right)}\left(\mathbb{R}^{d}\right)$, consisting of all functions $\varphi$ from $\mathscr{E}\left(\mathbb{R}^{d}\right)$ with support contained in $K$ such that

$$
\|\varphi\|_{K, h}:=\sup _{k \in \mathbb{N}_{0}^{d}} \sup _{x \in K} \frac{\left|\varphi^{(k)}(x)\right|}{h^{k} M_{k}}<\infty,
$$

with the topology induced by the above norm. Then the basic space $\mathscr{D}^{\left(M_{p}\right)}\left(\mathbb{R}^{d}\right)$ of test functions is defined by means of the projective and inductive limits as follows:

$$
\mathscr{D}^{\left(M_{p}\right)}\left(\mathbb{R}^{d}\right):=\underset{K \subset \subset \mathbb{R}^{d}}{\operatorname{ind} \lim } \underset{h \rightarrow 0}{\lim } \mathscr{D}_{K, h}^{\left(M_{p}\right)}\left(\mathbb{R}^{d}\right),
$$

where the symbol $K \subset \subset \mathbb{R}^{d}$ means that $K$ are regular compact sets growing up to $\mathbb{R}^{d}$.

In addition, for a fixed $m>0$, we denote by $\mathcal{S}_{2}^{\left(M_{p}\right), m}\left(\mathbb{R}^{d}\right)$ the space of all $C^{\infty}$ functions $\varphi$ such that

$$
\sigma_{m, 2}(\varphi):=\left[\sum_{\alpha, \beta \in \mathbb{N}_{0}^{d}}\left(\frac{m^{\alpha+\beta}}{M_{\alpha} M_{\beta}}\left\|\langle\cdot\rangle^{\beta} \varphi^{(\alpha)}\right\|_{2}\right)^{2}\right]^{1 / 2}<\infty,
$$

where the symbol $\langle\cdot\rangle$ is defined in (1), equipped with the topology induced by the above norm $\sigma_{m, 2}$. Then we define

$$
\mathcal{S}^{\left(M_{p}\right)}\left(\mathbb{R}^{d}\right):=\underset{m \rightarrow \infty}{\operatorname{proj}} \lim \mathcal{S}_{2}^{\left(M_{p}\right), m}\left(\mathbb{R}^{d}\right) .
$$


The strong dual of $\mathscr{D}^{\left(M_{p}\right)}\left(\mathbb{R}^{d}\right)$, denoted by $\mathscr{D}^{\prime\left(M_{p}\right)}\left(\mathbb{R}^{d}\right)$, is called the space of Beurling ultradistributions.

The following structural theorem (see [10], p. 76) says that Beurling ultradistributions are locally infinite derivatives of measures (continuous functions).

Theorem 7. Let $S \in \mathscr{D}^{\prime\left(M_{p}\right)}\left(\mathbb{R}^{d}\right)$. Then, for each open, relatively compact set $U$ in $\mathbb{R}^{d}$, there are measures $\mu_{\alpha} \in \mathscr{C}^{\prime}(\bar{U})$ for $\alpha \in \mathbb{N}_{0}^{d}$ and positive constants $L$ and $c$ such that

$$
\left.S\right|_{U}=\sum_{\alpha \in \mathbb{N}_{0}^{d}} \mu_{\alpha}^{(\alpha)}, \quad\left\|S_{\alpha}\right\|_{\mathscr{C}^{\prime}(\bar{U})} \leq \frac{c L^{\alpha}}{M_{\alpha}} \quad \text { for } \alpha \in \mathbb{N}_{0}^{d} .
$$

The space $\mathcal{S}^{\prime\left(M_{p}\right)}\left(\mathbb{R}^{d}\right)$ of all Beurling tempered ultradistributions is meant as the strong dual of the space $\mathcal{S}^{\left(M_{p}\right)}\left(\mathbb{R}^{d}\right)$ defined previously; it was introduced by Pilipović in [73] (see also $[18,52,59])$. Since $\mathscr{D}^{\left(M_{p}\right)}\left(\mathbb{R}^{d}\right)$ is dense in $\mathcal{S}^{\left(M_{p}\right)}\left(\mathbb{R}^{d}\right)$ and the inclusion mapping is continuous, we have $\mathcal{S}^{\prime\left(M_{p}\right)}\left(\mathbb{R}^{d}\right) \subset$ $\mathscr{D}^{\prime\left(M_{p}\right)}\left(\mathbb{R}^{d}\right)$.

For more details concerning the definitions and properties of the aforementioned spaces of test functions and the spaces $\mathscr{D}^{\prime\left(M_{p}\right)}$ of Beurling ultradistributions and $\mathcal{S}^{\prime\left(M_{p}\right)}$ of Beurling tempered ultradistributions we refer to $[18,51,52$, $59,60,73-75]$.

The following result is a representation theorem for Beurling tempered ultradistributions (see [59]).

Theorem 8. Suppose that $S \in \mathcal{S}^{\prime\left(M_{p}\right)}\left(\mathbb{R}^{d}\right)$. Then there are functions $F_{\alpha}$ in $L^{2}\left(\mathbb{R}^{d}\right)$ for $\alpha \in \mathbb{N}_{0}^{d}$ and positive constants $\lambda$, $L$, and $c$ such that

$$
\left.S\right|_{U}=\sum_{\alpha \in \mathbb{N}_{0}^{d}}\left(E_{\lambda} F_{\alpha}\right)^{(\alpha)}, \quad\left\|F_{\alpha}\right\|_{2} \leq \frac{c L^{\alpha}}{M_{\alpha}} \quad \text { for } \alpha \in \mathbb{N}_{0}^{d},
$$

where $E_{\lambda}(x)=e^{M(\lambda|x|)}$ for $x \in \mathbb{R}^{d}$.

There are various general definitions of the convolution in $\mathscr{D}^{\prime\left(M_{p}\right)}$ of Beurling ultradistributions (see [51]) and of the convolution in $\mathcal{S}^{\prime\left(M_{p}\right)}$ of Beurling tempered ultradistributions (see [52]). They are suitable counterparts of the known general definitions of the convolution in $\mathscr{D}^{\prime}$ and the convolution in $\delta^{\prime}$ mentioned in Section 3. The fact that the mentioned definitions of the convolution in $\mathscr{D}^{\prime\left(M_{p}\right)}$ of Beurling ultradistributions are equivalent and that the corresponding definitions of the convolution in $\mathcal{S}^{\prime\left(M_{p}\right)}$ of Beurling tempered ultradistributions are equivalent was proved in [51, 52 ], respectively (see also [18]).

We will recall here only these definitions of the convolution in $\mathscr{D}^{\prime\left(M_{p}\right)}$ and in $\mathcal{S}^{\prime\left(M_{p}\right)}$ which correspond to Vladimirov's definition of the convolution in $\mathscr{D}^{\prime}$ and in $\mathcal{S}^{\prime}$, respectively. The definitions are based on the notions of strong $\mathscr{D}^{\left(M_{p}\right)}$-approximate unit and strong $\mathcal{S}^{\left(M_{p}\right)}$ approximate unit.

Definition 9. A sequence $\left(\eta_{n}\right)$ of elements of $\mathscr{D}^{\left(M_{p}\right)}\left(\mathbb{R}^{d}\right)$ is said to be a strong $\mathscr{D}^{\left(M_{p}\right)}$-approximate unit on $\mathbb{R}^{d}$ if for every $K \sqsubset \mathbb{R}^{d}$ there exists an $n_{0} \in \mathbb{N}$ such that $\eta_{n}(x)=1$ for $x \in K$ and $n \geq n_{0}$ (hence $\eta_{n} \rightarrow 1$ in $\mathscr{E}^{\left(M_{p}\right)}\left(\mathbb{R}^{d}\right)$ as $n \rightarrow \infty$ ) and, in addition, if there exists a positive constant $h$ such that

$$
\sup _{n \in \mathbb{N}} \sup _{k \in \mathbb{N}_{0}^{d}}\left(\frac{h^{k}}{M_{k}}\left\|\eta_{n}^{(k)}\right\|_{\infty}\right)<\infty
$$

One denotes the set of all strong $\mathscr{D}^{\left(M_{p}\right)}$-approximate units on $\mathbb{R}^{d}$ by $\overline{\mathbb{U}}^{\left(M_{p}\right)}\left(\mathbb{R}^{d}\right)$.

Definition 10. If in the previous definition the assumption that $\eta_{n} \in \mathscr{D}^{\left(M_{p}\right)}\left(\mathbb{R}^{d}\right)$ for $n \in \mathbb{N}$ is replaced by $\eta_{n} \in \mathcal{S}^{\left(M_{p}\right)}\left(\mathbb{R}^{d}\right)$ for $n \in \mathbb{N}$ and the remaining assumptions are preserved, then the sequence $\left(\eta_{n}\right)$ is called a strong $\mathcal{S}^{\left(M_{p}\right)}$-approximate unit. One denotes the set of all strong $\mathcal{S}^{\left(M_{p}\right)}$-approximate units on $\mathbb{R}^{d}$ by $\overline{\mathbb{U}}_{s}^{\left(M_{p}\right)}\left(\mathbb{R}^{d}\right)$.

Remark 11. According to the known Denjoy-Carleman-Mandelbrojt theorem, the previously defined class $\overline{\mathbb{U}}^{\left(M_{p}\right)}\left(\mathbb{R}^{d}\right)$ of strong $\mathscr{D}^{\left(M_{p}\right)}$-approximate units as well as the class $\overline{\mathbb{U}}_{s}^{\left(M_{p}\right)}\left(\mathbb{R}^{d}\right)$ of strong $\mathcal{S}^{\left(M_{p}\right)}$-approximate units contains sufficiently many sequences, for example, all sequences of the form

$$
\eta_{n}(x)=\eta\left(\frac{x}{n}\right), \quad x \in \mathbb{R}^{d}, n \in \mathbb{N}
$$

where $\eta$ is a function of class $\mathscr{D}^{\left(M_{p}\right)}\left(\mathbb{R}^{d}\right)$ or $\delta^{\left(M_{p}\right)}\left(\mathbb{R}^{d}\right)$, respectively, such that $\eta=1$ in some neighbourhood of 0 .

Vladimirov's version of the definition of the convolution in $\mathscr{D}^{\prime\left(M_{p}\right)}$ of Beurling ultradistributions has the following form.

Definition 12. For given Beurling ultradistributions $S, T$ $\in \mathscr{D}^{\prime\left(M_{p}\right)}\left(\mathbb{R}^{d}\right)$ the convolution $S * T$ in $\mathscr{D}^{\prime\left(M_{p}\right)}$ is defined by

$$
\langle S * T, \varphi\rangle:=\lim _{n \rightarrow \infty}\left\langle S \otimes T, \eta_{n} \varphi^{\Delta}\right\rangle, \quad \varphi \in \mathscr{D}^{\left(M_{p}\right)}\left(\mathbb{R}^{d}\right),
$$

whenever the limit in (26) exists for every strong approximate unit $\left(\eta_{n}\right) \in \overline{\mathbb{U}}^{\left(M_{p}\right)}\left(\mathbb{R}^{2 d}\right)$ and $\varphi \in \mathscr{D}^{\left(M_{p}\right)}\left(\mathbb{R}^{d}\right)$.

Analogously, the convolution in $\mathcal{S}^{\prime\left(M_{p}\right)}$ of Beurling tempered ultradistributions can be defined as follows.

Definition 13. For given two Beurling tempered ultradistributions $S, T \in \mathcal{S}^{\prime\left(M_{p}\right)}\left(\mathbb{R}^{d}\right)$ we define the convolution $S * T$ in $\mathcal{S}^{\prime\left(M_{p}\right)}$ by

$$
\langle S * T, \psi\rangle:=\lim _{n \rightarrow \infty}\left\langle S \otimes T, \eta_{n} \psi^{\Delta}\right\rangle, \quad \psi \in \mathcal{S}^{\left(M_{p}\right)}\left(\mathbb{R}^{d}\right),
$$

whenever the limit in (27) exists for every strong approximate unit $\left(\eta_{n}\right) \in \overline{\mathbb{U}}_{s}^{\left(M_{p}\right)}\left(\mathbb{R}^{2 d}\right)$ and $\psi \in \mathcal{S}^{\left(M_{p}\right)}\left(\mathbb{R}^{d}\right)$. 


\section{Compatibility Conditions}

Let us present various forms of the condition imposed on sets $A, B \subseteq \mathbb{R}^{d}$ in the context of the convolution of distributions (see [5, p. 383]; see also $[6,7]$ ). They can be expressed in a shorter way by means of the notation introduced in (3).

Let us begin by recalling known equivalent forms of the condition in case the considered sets $A$ and $B$ are closed in $\mathbb{R}^{d}$ (see, e.g., $[5$, p. 383]).

Proposition 14. Let $A, B \subseteq \mathbb{R}^{d}$ be arbitrary closed sets. The following conditions are equivalent:

$$
\begin{aligned}
& (\Sigma)(A \times B) \cap K^{\triangle} \sqsubset \mathbb{R}^{2 d} \text { for every } K \sqsubset \mathbb{R}^{d} ; \\
& \left(\Sigma^{\prime}\right) A \cap(K-B) \sqsubset \mathbb{R}^{d} \text { for every } K \sqsubset \mathbb{R}^{d} ; \\
& \left(\Sigma^{\prime \prime}\right)(K-A) \cap B \sqsubset \mathbb{R}^{d} \text { for every } K \sqsubset \mathbb{R}^{d} .
\end{aligned}
$$

The meaning of the conditions for $d=1$ can be seen in Figure 1 (where $X$ and $Y$ mean $\mathbb{R}^{d}$ ).

As proved in $\left[5\right.$, pp. 383-384], conditions $(\Sigma)-\left(\Sigma^{\prime \prime}\right)$ are equivalent if the sets $A$ and $B$ are closed in $\mathbb{R}^{d}$. In general, we have the following equivalence (see [76]).

Proposition 15. Let $A, B \subseteq \mathbb{R}^{d}$ be arbitrary sets. The following conditions are equivalent:

$\left(\Sigma_{b}\right)(A \times B) \cap K^{\Delta}$ is bounded in $\mathbb{R}^{2 d}$ for every bounded set $K$ in $\mathbb{R}^{d}$;

$\left(\Sigma_{b}^{\prime}\right) A \cap(K-B)$ is bounded in $\mathbb{R}^{d}$ for every bounded set $K$ in $\mathbb{R}^{d}$;

$\left(\Sigma_{b}^{\prime \prime}\right)(K-A) \cap B$ is bounded in $\mathbb{R}^{d}$ for every bounded set $K$ in $\mathbb{R}^{d}$;

$(M)$ if $x_{n} \in A$ and $y_{n} \in B$ for $n \in \mathbb{N}$, then $\lim _{n \rightarrow \infty}\left(\left|x_{n}\right|+\right.$ $\left.\left|y_{n}\right|\right)=\infty$ implies $\lim _{n \rightarrow \infty}\left|x_{n}+y_{n}\right|=\infty$.

If the sets $A, B \subseteq \mathbb{R}^{d}$ are closed, then each of the above conditions is equivalent to each of conditions $(\Sigma)-\left(\Sigma^{\prime \prime}\right)$.

Definition 16. Two sets $A, B \subseteq \mathbb{R}^{d}$ are called compatible if one of equivalent conditions $\left(\Sigma_{b}\right)-(M)$ is satisfied.

Remark 17. The condition in the form $(M)$ and the name "compatible sets" were introduced in [54] (see also [7, p. 124127]).

There are two well known particular cases of compatible sets $A$ and $B$ in $\mathbb{R}^{1}$ :

$\left(1^{\circ}\right)$ at least one of the sets $A$ and $B$ is bounded;

$\left(2^{\circ}\right)$ both $A$ and $B$ are bounded from the left or both from the right.

Case $\left(1^{\circ}\right)$ extends clearly to $\mathbb{R}^{d}$ for $d>1$ and case $\left(2^{\circ}\right)$ can be described in $\mathbb{R}^{d}$ in the following form: $A, B \subset \mathbb{R}^{d}$ are (or are contained in) suitable cones with vertices at 0 such that $A$ is an open convex cone and $B \subset A^{*}$, where $A^{*}$ means the cone dual to $A$ (see [18, pp. 4-6]; [7, pp. 129-130]; [6, pp.

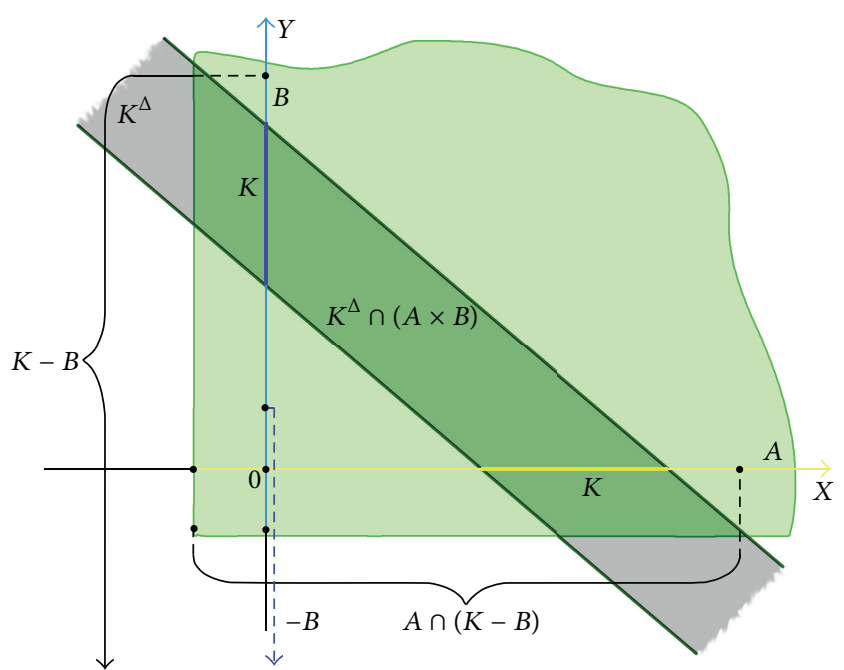

Figure 1: Compatible sets.

63-64]). For $d=2$, case $\left(2^{\circ}\right)$ is illustrated in Figure 2 (where $X_{1}$ and $X_{2}$ mean $\mathbb{R}^{1}$ ).

Another, less obvious, case of compatible sets in $\mathbb{R}^{1}$ is the following:

$\left(3^{\circ}\right)$ both sets $A$ and $B$ in $\mathbb{R}^{1}$ are unbounded from both sides, that is, unbounded both from below and from above in $\mathbb{R}^{1}$.

In [63] (see also [56-58]) various pairs of compatible sets $A$ and $B$ in $\mathbb{R}^{1}$, both unbounded from both sides, are considered; for $A=B$ in particular. They were constructed as countable sums of intervals (of length 1) situated in $\mathbb{R}^{1}$ in a specific way. A general idea lies in constructing a sequence of intervals on the positive half-line (with suitable distances between them) and in shifting in a proper way their symmetric counterparts on the negative half-line. Let $\left(p_{n}\right)_{n \in \mathbb{N}_{0}}$ be a sequence of positive integers. First denote the sets $P_{n}$ of indices $P_{n}:=\left\{0,1, \ldots, p_{n}-1\right\}$ for $n \in \mathbb{N}_{0}$. Consider the (doubly indexed) intervals $I_{n, k}$ and $J_{n, k}$ in $(-\infty, 0)$ and $(0, \infty)$, respectively, defined in the following way:

$$
I_{n, k}:=\left[a_{n, k}, a_{n, k}+1\right), \quad J_{n, k}:=\left(b_{n, k}, b_{n, k}+1\right],
$$

where

$$
\begin{gathered}
a_{n, k}:=-\sum_{j=0}^{n-1}(2 j+1) p_{j}-(2 n+1) k-(n+1), \\
b_{n, k}:=\sum_{j=0}^{n-1}(2 j+1) p_{j}+(2 n+1) k
\end{gathered}
$$

for any $k \in P_{n}$ and $n \in \mathbb{N}_{0}$ (the first sum on the right in (29) and (30) is meant to be equal to 0 for $n=0)$. Now define the set $A \subset \mathbb{R}^{1}$ by

$$
A:=\bigcup_{n \in \mathbb{N}_{0}}\left(A_{n} \cup B_{n}\right), \quad \text { where } A_{n}:=\bigcup_{k \in P_{n}} I_{n, k}, B_{n}:=\bigcup_{k \in P_{n}} J_{n, k} .
$$




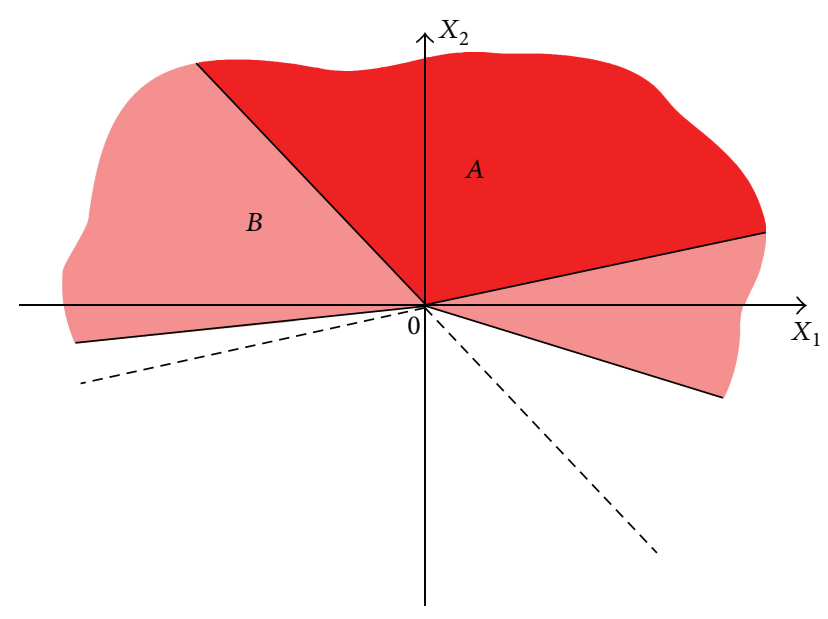

Figure 2: Cones.

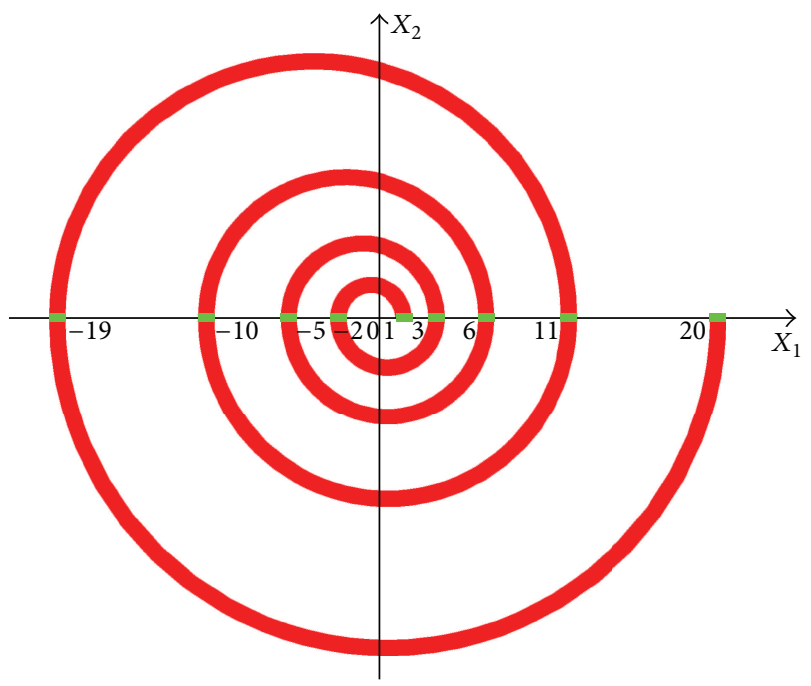

FIgURE 3: Spiral.

\section{Compatibility of Support and Existence of Convolution}

The condition of compatibility (see Definition 16) of the supports of locally integrable functions is sufficient for existence of their convolution in $L_{\text {loc }}^{1}$ (see, e.g., [7, p. 124]; see also $[5,6]$ ).

Theorem 18. Let $F, G \in L_{\mathrm{loc}}^{1}\left(\mathbb{R}^{d}\right)$. If the supports of the functions $F$ and $G$ are compatible sets, then the convolution $F * G$ exists in $L_{\text {loc }}^{1}$.

The condition of compatibility of supports is also sufficient for existence of the convolution in the space $\mathscr{D}^{\prime}\left(\mathbb{R}^{d}\right)$ of distributions. The following result is very well known (see, e.g., $[7$, p. 156]; see also $[5,6])$.

Theorem 19. Let $S, T \in \mathscr{D}^{\prime}\left(\mathbb{R}^{d}\right)$. If the supports of the distributions $S$ and $T$ are compatible sets, then $S * T$ exists in $\mathscr{D}^{\prime}$ and $S * T \in \mathscr{D}^{\prime}\left(\mathbb{R}^{d}\right)$.

However, it follows from Theorem 3 that the counterpart of Theorem 19 for the convolution in $\mathcal{S}^{\prime}$ of two tempered distributions is not true under the assumption of compatibility of their supports. In particular, the compatibility of supports of two functions of the class $\mathscr{P}\left(\mathbb{R}^{d}\right)$ does not guarantee that their convolution belongs to this class. This requires a suitable modification of the notion. In [56-58], the following modification of compatibility was introduced.

Definition 20. Two sets $A, B \subseteq \mathbb{R}^{d}$ are polynomially compatible, if there is a positive polynomial $P$ on $[0, \infty)$ such that the following implication holds:

$$
x \in A, y \in B \Longrightarrow|x|+|y| \leq P(|x+y|) .
$$

The assumption of polynomial compatibility of supports is sufficient for the convolution of two measurable slowly increasing functions to exist in $L_{\mathrm{loc}}^{1}$ and to represent a function of the same class (see [56-58]):

Theorem 21. Let $F, G \in \mathscr{P}\left(\mathbb{R}^{d}\right)$. If the supports of the functions $F$ and $G$ are polynomially compatible sets, then $F * G$ exists in $L_{\text {loc }}^{1}$ and $F * G \in \mathscr{P}\left(\mathbb{R}^{d}\right)$.

Under the assumption of polynomial compatibility of supports the result concerning the convolution in $\mathcal{S}^{\prime}$ is analogous to Theorem 19. Namely, the condition is sufficient for existence of the convolution of two tempered distributions in $\mathcal{S}^{\prime}[56,57]$ (see also $\left.[50,58]\right)$.

Theorem 22. Let $S, T \in \mathcal{S}^{\prime}\left(\mathbb{R}^{d}\right)$. If the supports of the tempered distributions $S$ and $T$ are polynomially compatible sets, then the convolution $S * T$ exists in $\mathcal{S}^{\prime}$ and $S * T \in \mathcal{S}^{\prime}\left(\mathbb{R}^{d}\right)$.

The last result was extended in different ways to the case of distributions in the Gelfand-Shilov spaces $\mathscr{K}^{\prime}\left(M_{p}\right)$ in $[60-$ 62] (see also [76]).

There exist results on the existence of the convolution in $\mathscr{D}^{\prime\left(M_{p}\right)}$ of Beurling ultradistributions and of the convolution 
in $\mathcal{S}^{\prime\left(M_{p}\right)}$ of Beurling tempered ultradistributions, analogous to Theorems 19 and 22. Let us recall the following existence result concerning the convolution in $\mathscr{D}^{\prime\left(M_{p}\right)}$ of Beurling ultradistributions, proved in [59].

Theorem 23. Let $S, T \in \mathscr{D}^{\prime\left(M_{p}\right)}\left(\mathbb{R}^{d}\right)$. If the supports of the Beurling ultradistributions $S$ and $T$ are compatible sets, then the convolution $S * T$ exists in $\mathscr{D}^{\prime\left(M_{p}\right)}$ and $S * T \in \mathscr{D}^{\prime\left(M_{p}\right)}\left(\mathbb{R}^{d}\right)$.

In [59], a modification of compatibility condition corresponding to the space $\mathcal{S}^{\prime\left(M_{p}\right)}$ of Beurling tempered ultradistributions was introduced via the associated function $M$ for the sequence $\left(M_{p}\right)$. We present it below in a slightly relaxed form (see [77]).

Definition 24. Two sets $A, B \subseteq \mathbb{R}^{d}$ are $M$-compatible, if there is a constant $\theta>0$ such that $M(|x|)+M(|y|) \leq M(\theta|x+y|)+\theta$ for all $x \in A$ and $y \in B$.

The following theorem is true (compare [59] and [77]).

Theorem 25. Let $S, T \in \mathcal{S}^{\prime\left(M_{p}\right)}\left(\mathbb{R}^{d}\right)$. If the supports the Beurling tempered ultradistributions $S$ and $T$ are $M$-compatible sets, then the convolution $S * T$ exists in $\mathcal{S}^{\prime\left(M_{p}\right)}$ and $S * T \in$ $\mathcal{S}^{\prime\left(M_{p}\right)}\left(\mathbb{R}^{d}\right)$.

\section{Inverse Results}

It should be noted that the conditions of compatibility and polynomial compatibility of supports of distributions and tempered distributions, assumed in Theorems 19 and 22, are in a sense optimal in terms of supports for the existence of the convolutions in $\mathscr{D}^{\prime}$ and in $\mathcal{S}^{\prime}$, respectively, and they cannot be relaxed. The situation is precisely described by Theorems 26 and 27 formulated below which were proved in [78].

Theorem 26. Let $A, B \subseteq \mathbb{R}^{d}$. Suppose that the convolution $S * T$ exists in $\mathscr{D}^{\prime}$ for each pair $S, T \in \mathscr{D}^{\prime}\left(\mathbb{R}^{d}\right)$ of distributions such that supp $S \subseteq A$ and supp $T \subseteq B$. Then $A$ and $B$ are compatible.

Theorem 27. Let $A, B \subseteq \mathbb{R}^{d}$. Suppose that the convolution $S * T$ exists in $S^{\prime}$ for each pair $S, T \in \mathcal{S}^{\prime}\left(\mathbb{R}^{d}\right)$ of tempered distributions such that supp $S \subseteq A$ and supp $T \subseteq B$. Then $A$ and $B$ are polynomially compatible.

We prove next an analogue of Theorem 26 for the case of Beurling ultradistributions.

Theorem 28. Let $A, B \subseteq \mathbb{R}^{d}$. Assume that the convolution $S * T$ exists in $\mathscr{D}^{\prime\left(M_{p}\right)}$ for each pair $S, T \in \mathscr{D}^{\prime\left(M_{p}\right)}\left(\mathbb{R}^{d}\right)$ of Beurling ultradistributions with the supports contained in $A$ and $B$, respectively. Then $A$ and $B$ are compatible.

Proof. Assume that $A$ and $B$ are not compatible. This assumption implies that there exist sequences $\left(x_{n}\right)$ and $\left(y_{n}\right)$ of elements of sets $A$ and $B$, respectively, such that

$$
\begin{array}{r}
\lim _{n \rightarrow \infty}\left|x_{n}\right|=\lim _{n \rightarrow \infty}\left|y_{n}\right|=\infty, \quad \lim _{n \rightarrow \infty}\left(x_{n}+y_{n}\right)=z \\
\text { in } \mathbb{R}^{d} \text { for some } z \in \mathbb{R}^{d} .
\end{array}
$$

It is clear that we may assume, in addition, that

$$
\begin{array}{r}
\left|x_{n+1}\right|-\left|x_{n}\right|>1, \\
\left|y_{n+1}\right|-\left|y_{n}\right|>1, \\
\left|x_{n}+y_{n}-z\right|<1, \\
\text { for } n \in \mathbb{N} .
\end{array}
$$

Let us define $S, T \in \mathscr{D}^{\prime\left(M_{p}\right)}\left(\mathbb{R}^{d}\right)$ as measures of the form

$$
S:=\sum_{i=1}^{\infty} \delta_{x_{i}}, \quad T:=\sum_{i=1}^{\infty} \delta_{y_{i}},
$$

where $\delta_{x}$ denotes the Dirac measure in $\mathscr{D}^{\prime\left(M_{p}\right)}\left(\mathbb{R}^{d}\right)$ concentrated at the point $x \in \mathbb{R}^{d}$, that is, the Beurling ultradistribution defined by $\left\langle\delta_{x}, \varphi\right\rangle:=\varphi(x)$ for all $\varphi \in$ $\mathscr{D}^{\left(M_{p}\right)}\left(\mathbb{R}^{d}\right)$. Obviously, supports of $S$ and $T$ are contained in $A$ and $B$, respectively.

Choose nonnegative functions $\eta \in \mathscr{D}^{\left(M_{p}\right)}\left(\mathbb{R}^{2 d}\right)$ and $\omega \epsilon$ $\mathscr{D}^{\left(M_{p}\right)}\left(\mathbb{R}^{d}\right)$, identically equal to 1 on the balls $\bar{B}_{2 d}(0, \sqrt{2})$ and $\bar{B}_{d}(z, 1)$, respectively. Then define the functions $\eta_{k}$ by

$$
\eta_{k}(x, y):=\eta\left(\frac{x}{k}, \frac{y}{k}\right) \quad \text { for } x, y \in \mathbb{R}^{d}, k \in \mathbb{N} .
$$

Hence

$$
\begin{gathered}
\omega(u)=1 \quad \text { if }|u-z| \leq 1, \\
\eta_{k}(x, y)=1 \quad \text { if }|x| \leq k,|y| \leq k,
\end{gathered}
$$

for $x, y$, and $u \in \mathbb{R}^{d}$ and $k \in \mathbb{N}$. Clearly, $\left(\eta_{k}\right) \in \overline{\mathbb{U}}\left(\mathbb{R}^{d}\right)$.

For sufficiently large $k$, we have the inclusion $\bar{B}_{d}(z, 1) \subset$ $\bar{B}_{d}(0, k)$. For such indices $k$, due to (34)-(37), we get

$$
\left\langle S \otimes T, \eta_{k} \omega^{\Delta}\right\rangle \geq \sum_{i=1}^{\infty} \eta_{k}\left(x_{i}, y_{i}\right) \omega\left(x_{i}+y_{i}\right) \geq s_{k},
$$

where $s_{k}$ is the number of the indices $i$ for which $x_{i}, y_{i} \in$ $\bar{B}_{d}(0, k)$. Since $s_{k} \rightarrow \infty$ as $k \rightarrow \infty$, we conclude that the convolution $S * T$ does not exist in $\mathscr{D}^{\prime\left(M_{p}\right)}$, which contradicts the assumption. Thus the assertion of the theorem is proved.

Whether Theorem 27 has its counterpart for Beurling tempered ultradistributions remains an open problem.

Problem 29. Let $A, B \subseteq \mathbb{R}^{d}$. Assume that the convolution $S * T$ exists in $\mathcal{S}^{\prime\left(M_{p}\right)}$ for each pair $S, T \in \mathcal{S}^{\prime\left(M_{p}\right)}\left(\mathbb{R}^{d}\right)$ of Beurling ultradistributions with the supports contained in $A$ and $B$, respectively. Do the sets $A$ and $B$ have to be $M$-compatible?

Let us return now to the case of the convolution in $L_{\text {loc }}^{1}$ of locally integrable functions. We will modify for this case the notion of compatible sets expressed by means of certain equivalent conditions (cf. [79]). 
Proposition 30. Let $A$ and $B$ be measurable sets in $\mathbb{R}^{d}$. The following conditions are equivalent.

(i) For each $K \sqsubset \mathbb{R}^{d}$ there exists an $L \sqsubset \mathbb{R}^{d}$ such that $\ell^{d}\left((x-A) \cap B \cap L^{c}\right)=0$ for all $x \in K$.

(ii) For each $K \sqsubset \mathbb{R}^{d}$ there is an $L \sqsubset \mathbb{R}^{d}$ such that

$$
\ell^{d}\left(\left\{x \in K: \ell^{d}\left((x-A) \cap B \cap L^{c}\right)>0\right\}\right)=0 .
$$

(iii) For arbitrary sequences $\left(A_{n}\right)$ and $\left(B_{n}\right)$ of positively bounded subsets of $A$ and $B$, respectively, the following implication holds:

$\lim _{n \rightarrow \infty}\left(\left|A_{n}\right|_{\infty}+\left|B_{n}\right|_{\infty}\right)=\infty$ implies $\lim _{n \rightarrow \infty}\left|A_{n}+B_{n}\right|_{\infty}=\infty$.

(iv) For arbitrary sequences $\left(x_{n}\right)$ and $\left(y_{n}\right)$ of massive points of the sets $A$ and $B$, respectively, the following implication holds:

$$
\lim _{n \rightarrow \infty}\left(\left|x_{n}\right|+\left|y_{n}\right|\right)=\infty \text { implies } \lim _{n \rightarrow \infty}\left|x_{n}+y_{n}\right|=\infty \text {. }
$$

Definition 31. One calls the sets $A, B \subset \mathbb{R}^{d}$ quasi-compatible if one of conditions (i)-(iv) is satisfied.

It is clear that if measurable sets $A, B \subset \mathbb{R}^{d}$ are compatible, then they are quasi-compatible, but not conversely.

The proof of Proposition 30 is based on the two following lemmas (see [79]).

Lemma 32. If a measurable set $A \subset \mathbb{R}^{d}$ is positively bounded, then for every $\varepsilon$ with $0<\varepsilon<|A|_{\infty}$ there exists a massive point $x$ of $A$ such that $|x| \geq|A|_{\infty}-\varepsilon$.

Lemma 33. For any two measurable sets $A$ and $B$ in $\mathbb{R}^{d}$ such that $\ell^{d}(A)>0$ and $\ell^{d}(B)>0$ there exists a pair $(x, C)$, where $x$ is a point in $\mathbb{R}^{d}$ and $C$ is a measurable subset of $B$ with $\ell^{d}(C)>$ 0 , having the following property: for every $y \in C$ there exists a measurable subset $D_{y}$ of $B$ with $\ell^{d}\left(D_{y}\right)>0$ such that the inclusions $x+y-D_{y} \subseteq A$ hold for all $y \in C$.

The following assertion is an easy corollary from Theorem 18.

Theorem 34. If $F, G \in L_{\text {loc }}^{1}\left(\mathbb{R}^{d}\right)$ and the supports of the functions $F$ and $G$ are (contained in) quasi-compatible sets, then the convolution $F * G$ exists and represents a locally integrable function.

The condition of quasi-compatibility of measurable sets in $\mathbb{R}^{d}$ can be described in the following way in the context of the existence of the convolutions in the space $L_{\mathrm{loc}}^{1}\left(\mathbb{R}^{d}\right)$ of locally integrable functions (cf. [79]).

Theorem 35. Let $A$ and $B$ be measurable sets in $\mathbb{R}^{d}$. Suppose that convolution $F * G$ exists and represents a locally integrable function in $\mathbb{R}^{d}$ for any $F, G \in L_{\text {loc }}^{1}\left(\mathbb{R}^{d}\right)$ whose supports are contained in $A$ and $B$, respectively. Then $A$ and $B$ are quasicompatible.
Proof. Suppose that $A$ and $B$ are not quasi-compatible sets in $\mathbb{R}^{d}$. Then, by Proposition 30 , one can find two sequences $\left(x_{n}\right)$ and $\left(y_{n}\right)$ of massive points of the sets $A$ and $B$, respectively, such that

$$
\lim _{n \rightarrow \infty}\left|x_{n}\right|=\lim _{n \rightarrow \infty}\left|y_{n}\right|=\infty, \quad \rho:=\sup _{n \in \mathbb{N}}\left|x_{n}+y_{n}\right|<\infty .
$$

We may assume, in addition, that

$$
\left|x_{n+1}\right|-\left|x_{n}\right|>2, \quad\left|y_{n+1}\right|-\left|y_{n}\right|>2, \quad \text { for } n \in \mathbb{N} \text {. }
$$

Let $\bar{B}\left(x_{n}\right)$ and $\bar{B}\left(y_{n}\right)$ be the closed balls in $\mathbb{R}^{d}$ of radius 1 with centers at $x_{n}$ and $y_{n}$, respectively, for $n \in \mathbb{N}$. Let $\bar{C}$ be the closed ball with the center at 0 and radius $\rho+2$. Put

$$
A_{n}:=A \cap \bar{B}\left(x_{n}\right), \quad B_{n}:=B \cap \bar{B}\left(y_{n}\right), \quad \text { for } n \in \mathbb{N} .
$$

By (42), (43), and Proposition 30, we have $A_{n}+B_{n} \subseteq \bar{C}$ for all $n \in \mathbb{N}$ and, moreover, $A_{n} \cap A_{m}=\emptyset$ and $B_{n} \cap B_{m}=\emptyset$ for $n \neq m$; in addition, $\ell^{d}\left(A_{n}\right)>0$ and $\ell^{d}\left(B_{n}\right)>0$ for all $n \in \mathbb{N}$.

Define the functions $F, G$, and $\psi$ in the following way:

$$
F=\sum_{n=1}^{\infty} \alpha_{n} \chi_{A_{n}}, \quad G=\sum_{n=1}^{\infty} \beta_{n} \chi_{B_{n}}, \quad \psi=\chi_{\bar{C}},
$$

where $\alpha_{n}=\left[\ell^{d}\left(A_{n}\right)\right]^{-1}$ and $\beta_{n}=\left[\ell^{d}\left(B_{n}\right)\right]^{-1}$ for $n \epsilon$ $\mathbb{N}$. According to (44) and (43), $F$ and $G$ are well defined nonnegative locally integrable functions with the supports contained in $A$ and $B$, respectively. Moreover, $\psi \in L_{\text {loc }}^{1}$ and $\operatorname{supp} \psi=\bar{C} \sqsubset \mathbb{R}^{d}$. Hence

$$
\begin{aligned}
& \int_{\mathbb{R}^{d}}(|F| *|G|)(x) \psi(x) d x \\
& \quad \geq \sum_{n=1}^{\infty} \int_{A_{n}} \int_{B_{n}}\left[F(s) G(t) \chi_{\bar{C}}(s+t)\right] d s d t \\
& \quad \geq \sum_{n=1}^{\infty} \alpha_{n} \beta_{n} \int_{A_{n}} d s \int_{B_{n}} d t=\infty,
\end{aligned}
$$

which contradicts our assumption that $F * G$ exists in $L_{\text {loc }}^{1}$ and thus the proof of our assertion is completed.

\section{Acknowledgments}

The authors thank the referees for valuable comments which helped in improving the paper. Special thanks are addressed to Mr. K. Majchrowski for preparing the figures placed in the text. This work was partly supported by the Centre for Innovation and Transfer of Natural Sciences and Engineering Knowledge.

\section{References}

[1] L. Schwartz, Théorie des distributions, vol. 1-2, Hermann, Paris, France, 1950-1951.

[2] C. Chevalley, “Theory of Distributions," Lectures at Columbia University, 1950-1951. 
[3] I. M. Gelfand and G. E. Shilov, Generalized Functions, vol. 1, Academic Press, New York, NY, USA, 1964.

[4] I. M. Gelfand and G. E. Shilov, Generalized Functions, vol. 2, Academic Press, New York, NY, USA, 1968.

[5] J. Horváth, Topological Vector Spaces and Distributions, vol. 1, Addison-Wesley, London, UK, 1966.

[6] V. S. Vladimirov, Methods of the Theory of Generalized Functions, Analytical Methods and Special Functions, Taylor \& Francis, London, UK, 1st edition, 2002.

[7] P. Antosik, J. Mikusiński, and R. Sikorski, Theory of Distributions.The Sequential Approach, Państwowe Wydawnictwo Naukowe, Warsaw, Poland, 1973.

[8] C. Roumieu, "Sur quelques extension de la notion de distribution," Annales Scientifiques de l'École Normale Supérieure, vol. 77, pp. 41-121, 1960.

[9] A. Beurling, "Quasi-analycity and general distributions," Lectures 4 and 5, AMS, Summer Institute, Stanford, Calif, USA, 1961.

[10] H. Komatsu, "Ultradistributions I," Journal of Faculty of Science, University of Tokyo IA, vol. 20, pp. 25-105, 1973.

[11] H. Komatsu, "Ultradistributions II," Journal of Faculty of Science, University of Tokyo IA, vol. 24, pp. 607-628, 1977.

[12] H. Komatsu, "Ultradistributions III," Journal of Faculty of Science, University of Tokyo IA, vol. 29, pp. 653-718, 1982.

[13] I. Ciorănescu and L. Zsidó, “ $\omega$-ultradistributions and their application to operator theory," in Spectral Theory, vol. 8 of Banach Center Publications, pp. 77-220, Państwowe Wydawnictwo Naukowe, Warsaw, Poland, 1982.

[14] C. G. Bollini, T. Escobar, and M. C. Rocca, "Convolution of ultradistributions and field theory," International Journal of Theoretical Physics, vol. 38, no. 9, pp. 2315-2332, 1999.

[15] J. J. Betancor, C. Fernández, and A. Galbis, "Beurling ultradistributions of $L_{p}$-growth," Journal of Mathematical Analysis and Applications, vol. 279, no. 1, pp. 246-265, 2003.

[16] C. G. Bollini, P. Marchiano, and M. C. Rocca, "Convolution of ultradistributions, field theory, Lorentz invariance and resonances," International Journal of Theoretical Physics, vol. 46, no. 12, pp. 3030-3059, 2007.

[17] R. D. Carmichael, A. Kamiński, and S. Pilipović, "Cauchy and Poisson integrals of ultradistributions in $D^{\prime}\left(*, L^{s}\right)$," Integral Transforms and Special Functions, vol. 17, no. 2-3, pp. 135-139, 2006.

[18] R. D. Carmichael, A. Kamiński, and S. Pilipović, Boundary Values and Convolution in Ultradistribution Spaces, vol. 1 of Series on Analysis, Application and Computation, World Scientific Publishing, Hackensack, NJ, USA, 2007.

[19] M. Sato, “Theory of hyperfunctions I," Journal of Faculty of Science University of Tokyo, Section I, vol. 8, pp. 139-193, 1959.

[20] M. Sato, "Theory of hyperfunctions II," Journal of Faculty of Science University of Tokyo, Section I, vol. 8, pp. 387-437, 1960.

[21] A. Kaneko, Introduction to Hyperfunctions, vol. 3 of Mathematics and Its Applications, Kluwer, Dordrecht, Netherlands, 1988.

[22] J. Mikusiński, Rachunek Operatorów, Państwowe Wydawnictwo Naukowe, Warsaw, Poland, 1953.

[23] J. Mikusiński, Operational Calculus, Pergamon Press, New York, NY, USA; Państwowe Wydawnictwo Naukowe, Warsaw, Poland, 1st edition, 1959.

[24] J. Mikusiński, Operational Calculus, vol. 1 of 2nd Enlarged TwoVolume English Editions, Pergamon Press, New York, NY, USA, 2nd edition, 1983.
[25] J. Mikusiński and T. K. Boehme, Operational Calculus of 2nd Enlarged Two-Volume English Editions, vol. 2 of 2nd Enlarged Two-Volume English Editions, Pergamon Press, New York, NY, USA, 2nd edition, 1983.

[26] T. K. Boehme, “The support of Mikusiński operators," Transactions of the American Mathematical Society, vol. 176, pp. 319-334, 1973.

[27] J. Mikusiński and P. Mikusiński, "Quotients de suites et leurs applications dans l'analyse fonctionnelle," Comptes Rendus des Séances de l'Académie des Sciences. I Mathématiques, vol. 293, no. 9, pp. 463-464, 1981.

[28] P. Mikusiński, “Convergence of Boehmians," Japanese Journal of Mathematics, vol. 9, no. 1, pp. 159-179, 1983.

[29] J. Burzyk and P. Mikusiński, "A generalization of the construction of a field of quotients with applications in analysis," International Journal of Mathematical Sciences, vol. 2, no. 2, pp. 229-236, 2003.

[30] J. Burzyk, P. Mikusiński, and D. Nemzer, "Remarks on topological properties of Boehmians," The Rocky Mountain Journal of Mathematics, vol. 35, no. 3, pp. 727-740, 2005.

[31] D. Atanasiu, P. Mikusiński, and D. Nemzer, "An algebraic approach to tempered distributions," Journal of Mathematical Analysis and Applications, vol. 384, no. 2, pp. 307-319, 2011.

[32] J. J. Betancor, C. Jerez, S. M. Molina, and L. Rodríguez-Mesa, "Distributional convolutors for Fourier transform," Journal of Mathematical Analysis and Applications, vol. 325, no. 1, pp. 459468, 2007.

[33] J. Kisyński, "On Fourier transforms of distribution semigroups," Journal of Functional Analysis, vol. 242, no. 2, pp. 400-441, 2007.

[34] M. Kostić and S. Pilipović, "Global convoluted semigroups," Mathematische Nachrichten, vol. 280, no. 15, pp. 1727-1743, 2007.

[35] P. C. Kunstmann, M. Mijatović, and S. Pilipović, "Classes of distribution semigroups," Studia Mathematica, vol. 187, no. 1, pp. 37-58, 2008.

[36] J. Kisyński, "One-parameter semigroups in the convolution algebra of rapidly decreasing distributions," Colloquium Mathematicum, vol. 128, no. 1, pp. 49-68, 2012.

[37] M. Kostić and S. Pilipović, "Ultradistributions of semigroups," Sibirskiu Matematicheskiu Zhurnal, vol. 53, no. 2, pp. 291-305, 2012.

[38] J.-F. Colombeau, New Generalized Functions and Multiplication of Distributions, vol. 84, North-Holland, Amsterdam, Netherlands, 1984.

[39] H. A. Biagioni, A Nonlinear Theory of Generalized Functions, vol. 1421 of Lecture Notes in Mathematics, Springer, Berlin, Germany, 2nd edition, 1990.

[40] M. Nedeljkov, S. Pilipović, and D. Scarpalézos, The Linear Theory of Colombeau Generalized Functions, Addison Wesley, Boston, Mass, USA; Longman, Harlow, UK, 1998.

[41] S. Pilipović and D. Scarpalézos, "Colombeau generalized ultradistributions," Mathematical Proceedings of the Cambridge Philosophical Society, vol. 130, no. 3, pp. 541-553, 2001.

[42] M. Grosser, M. Kunzinger, M. Oberguggenberger, and R. Steinbauer, Geometric Theory of Generalized Functions with Applications to General Relativity, vol. 537 of Mathematics and Its Applications, Kluwer, Dordrecht, Netherlands, 2001.

[43] A. Delcroix, M. F. Hasler, S. Pilipović, and V. Valmorin, "Generalized function algebras as sequence space algebras," Proceedings of the American Mathematical Society, vol. 132, no. 7, pp. 2031-2038, 2004. 
[44] L. Schwartz, "Définitions integrale de la convolution de deux distributions," in Séminaire Schwartz, Année 1953-1954, Produits tensoriels topologiques et d'espaces vectoriels topologiques. Espaces vectoriels topologiques nucléaires. Applications, Expose $n^{\circ} 22$, Secrétariat Mathématique, Faculté de Sciences, Paris, France, 1954.

[45] R. Shiraishi, "On the definition of convolution for distributions," Journal of Science of the Hiroshima University. Series A, vol. 23, pp. 19-32, 1959.

[46] V. S. Vladimirov, Equations of Mathematical Physics, Marcel Dekker, New York, USA, 1971.

[47] J. Horváth, "Sur la convolution des distributions," Bulletin des Sciences Mathématiques, vol. 98, no. 3, pp. 183-192, 1974.

[48] B. Roider, "Sur la convolution des distributions," Bulletin des Sciences Mathématiques, vol. 100, pp. 193-199, 1974.

[49] P. Dierolf and J. Voigt, "Convolution and $S^{\prime}$-convolution of distributions," Collectanea Mathematica, vol. 29, no. 3, pp. 185-196, 1978.

[50] A. Kamiński, "Convolution, product and Fourier transform of distributions," Studia Mathematica, vol. 74, no. 1, pp. 83-96, 1982.

[51] A. Kamiński, D. Kovačević, and S. Pilipović, "The equivalence of various definitions of the convolution of ultradistributions," Trudy Matematicheskogo Instituta imeni V. A. Steklova, vol. 203, pp. 307-322, 1994.

[52] A. Kamiński, D. Perišić, and S. Pilipović, "On the convolution in the space of tempered ultradistributions of Beurling type," Integral Transforms and Special Functions, vol. 15, no. 4, pp. 323329, 2004.

[53] S. Pilipović and B. Prangoski, "Equivalence of several definitions of convolution of Roumieu ultradistributions," Monatshefte für Mathematik. In press.

[54] J. Mikusiński, "Sequential theory of the convolution of distributions," Studia Mathematica, vol. 29, pp. 151-160, 1968.

[55] J. Vindas and R. Estrada, "On the support of tempered distributions," Proceedings of the Edinburgh Mathematical Society.Series 2, vol. 53, no. 2, pp. 255-270, 2010.

[56] A. Kamiński, Całkowanie i operacje nieregularne [Ph.D. thesis], Institute of Mathematics, Polish Academy of Sciences, Warsaw, Poland, 1975.

[57] A. Kamiński, Operacje regularne i nieregularne na dystrybucjach [Preprint 11 of Seria B], Institute of Mathematics, Polish Academy of Sciences, Warsaw, Poland, 1981.

[58] A. Kamiński, "On convolutions, products and Fourier transforms of distributions," Bulletin de l'Académie Polonaise des Sciences, vol. 25, no. 4, pp. 369-374, 1977.

[59] A. Kamiński, D. Perišić, and S. Pilipović, "Existence theorems for convolution of ultradistributions," Dissertationes Mathematicae, vol. 340, pp. 79-91, 1995.

[60] S. Pilipović, "On the convolution in the space of $K^{\prime}\left\{M_{p}\right\}$-type," Mathematische Nachrichten, vol. 120, pp. 103-112, 1985.

[61] J. Uryga, On Some Criterion of Existance of the Convolution in the Space of Type $K\left\{M_{P}\right\}^{\prime}$ [Preprint 15 of Seria B], Institute of Mathematics, Polish Academy of Sciences, Warsaw, Poland, 1986.

[62] J. Uryga, "On compatiblity of supports of generalized functions of Gelfand-Shilov type," Bulletin of the Polish Academy of Sciences Mathematics, vol. 36, pp. 143-150, 1988.

[63] A. Kamiński and S. Mincheva-Kamińska, "Equivalent conditions for integrability and convolvability of distributions and tempered distributions".
[64] K. Yoshinaga and H. Ogata, "On the convolutions," Journal of Science of the Hiroshima University. Series A, vol. 22, pp. 15-24, 1958.

[65] Y. Hirata, "On convolutions in the theory of distributions," Journal of Science of the Hiroshima University. Series A, vol. 22, pp. 89-98, 1958.

[66] P. Wagner, “Zur faltung von distributionen," Mathematische Annalen, vol. 276, no. 3, pp. 467-485, 1987.

[67] R. Wawak, "Improper integrals of distributions," Studia Mathematica, vol. 86, pp. 205-220, 1987.

[68] N. Ortner, "On convolvability conditions for distributions," Monatshefte für Mathematik, vol. 160, no. 3, pp. 313-335, 2010.

[69] N. Ortner, "The Fourier exchange theorem for kernels," Applicable Analysis, vol. 90, no. 11, pp. 1635-1650, 2011.

[70] S. Mincheva-Kamińska, "Sequential approach to integrable distributions," Novi Sad Journal of Mathematics, vol. 41, no. 1, pp. 123-131, 2011.

[71] S. Mincheva-Kamińska, "Equivalence of sequential definitions of the convolution of distributions," Rendiconti del Seminario Matematico, vol. 69, no. 4, pp. 367-376, 2011.

[72] S. Mincheva-Kamińska, "Equivalent conditions for integrability and convolvability of distributions and tempered distributions".

[73] S. Pilipović, "Tempered ultradistributions," Unione Matematica Italiana Bollettino. B, vol. 2, no. 2, pp. 235-251, 1988.

[74] S. Pilipović, "On the convolution in the space of Beurling ultradistributions," Commentarii Mathematici Universitatis Sancti Pauli, vol. 40, no. 1, pp. 15-27, 1991.

[75] S. Pilipović, "Characterizations of bounded sets in spaces of ultradistributions," Proceedings of the American Mathematical Society, vol. 120, no. 4, pp. 1191-1206, 1994.

[76] A. Kamiński and J. Uryga, "Convolution in $K^{\prime}\left\{M_{p}\right\}$-spaces," in Generalized Functions, Convergence Structures, and Their Applications, pp. 187-196, Plenum Press, New York, NY, USA, 1988.

[77] A. Kamiński and S. Mincheva-Kaminska, "Existence theorems on convolution of functions, distributions and ultradistributions," in Traditional and Present-Day Topics in Real Analysis, pp. 39-54, Łódź University Press, , Łódź, Poland, 2013.

[78] A. Kamiński, "On the Rényi theory of conditional probabilities," Studia Mathematica, vol. 79, no. 2, pp. 151-191, 1984.

[79] A. Kamiński, "On almost compatible supports of locally integrable functions," Zeszyty Naukowe Politechniki Śląskiej, vol. 48, pp. 81-90, 1986. 


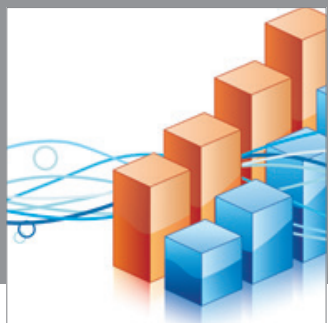

Advances in

Operations Research

mansans

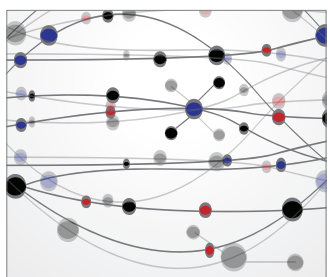

The Scientific World Journal
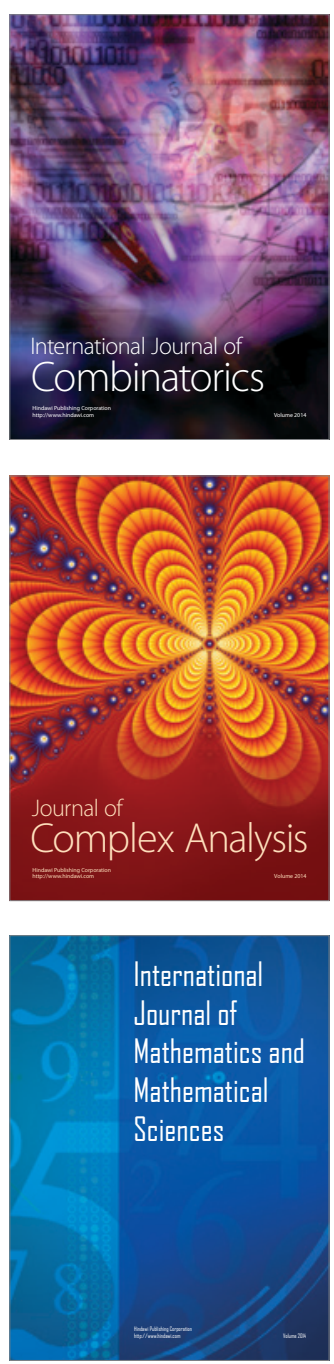
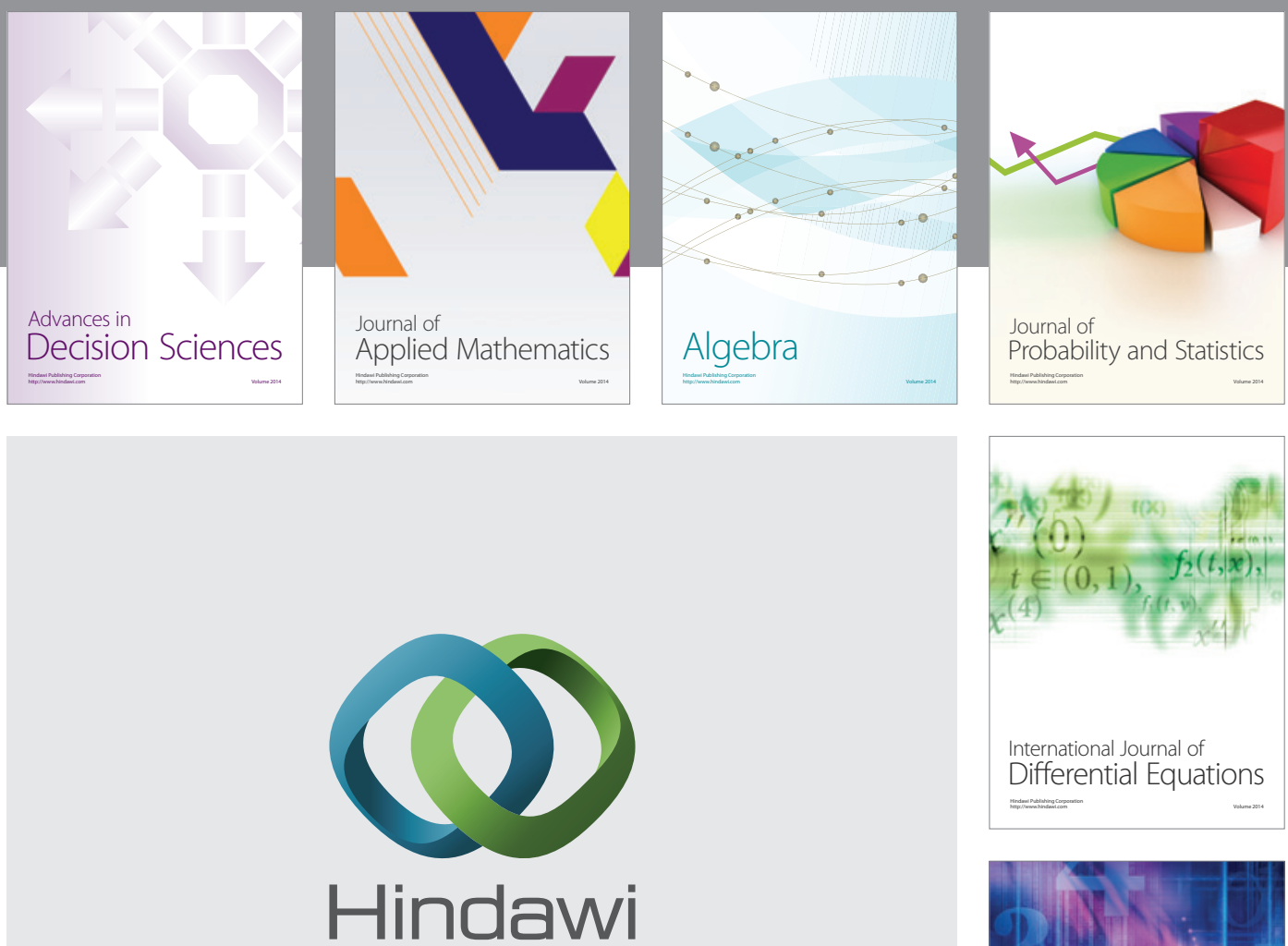

Submit your manuscripts at http://www.hindawi.com
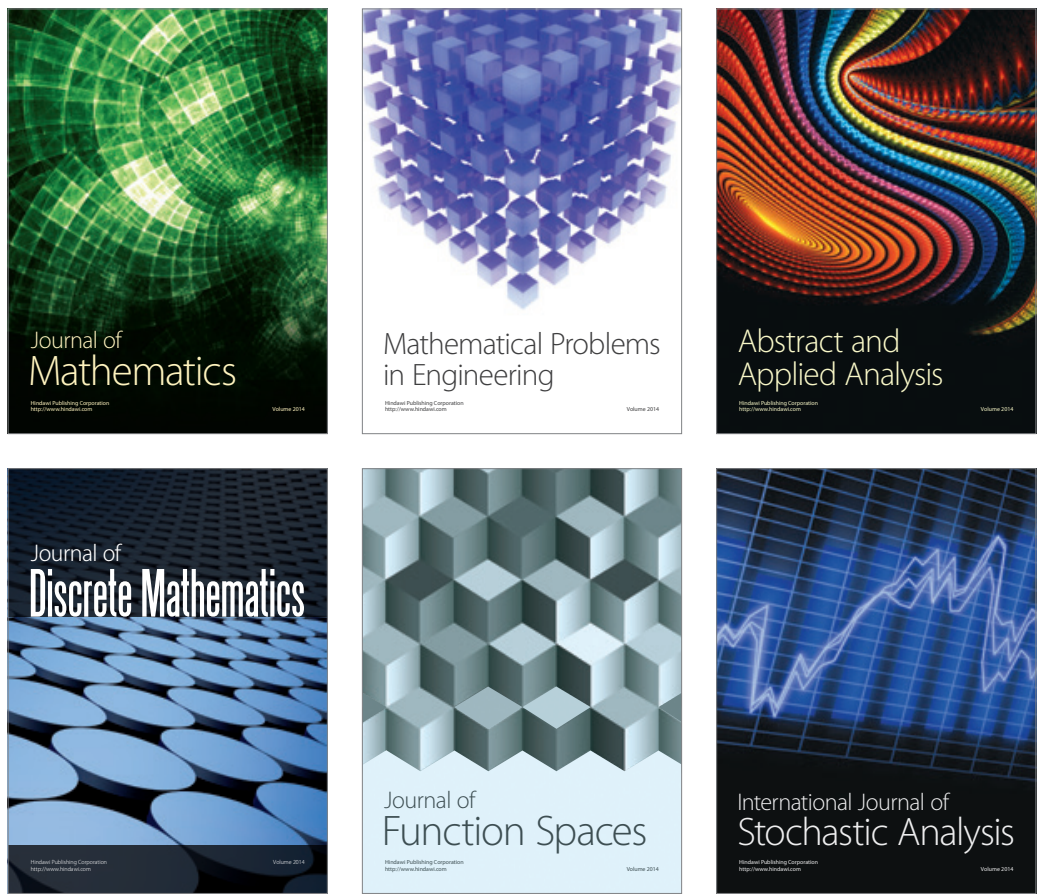

Journal of

Function Spaces

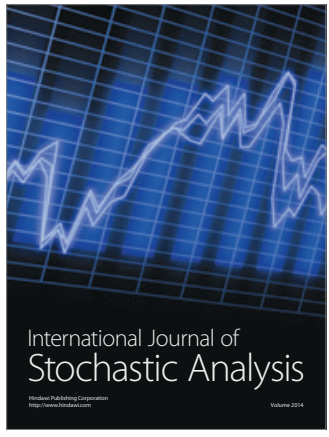

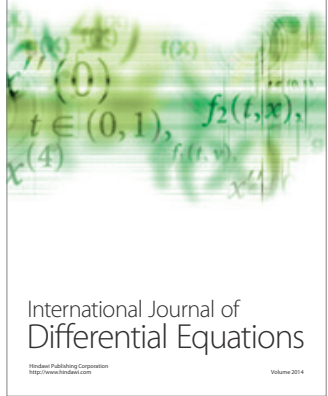
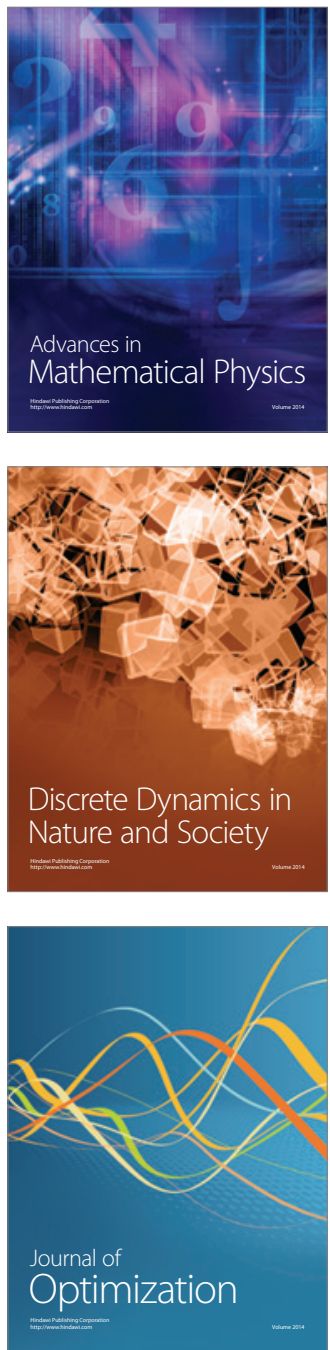